\title{
Strictly Nonblocking $f$-cast Photonic Switching Networks under General Crosstalk Constraints
}

\author{
Thanh-Nhan Nguyen, Hung Q. Ngo, Yang Wang \\ Computer Science and Engineering, \\ 201 Bell Hall, \\ State University of New York at Buffalo, \\ Amherst, NY 14260, USA. \\ \{nguyen9, hungngo, yw43\}@cse.buffalo.edu
}

\begin{abstract}
We derive conditions under which a $d$-ary multilog network is $f$-cast strictly nonblocking under the general crosstalk constraint that no active route can share more than $c$ switching elements with other active routes. The problem setting is very general and thus challenging in many ways: (1) $f$-cast covers both unicast $(f=1)$ and multicast/broadcast $(f=N)$, (2) $d$-ary networks are much more general then the commonly considered binary multilog networks, and (3) the crosstalk constraint covers both the usual link-blocking case $(c=n)$ and the crosstalk-free case $(c=0)$.

Prior to our work, results with the general crosstalk constraint are known only in the unicast case. Other results on strictly nonblocking $f$-cast networks are known without the general crosstalk constraint. Most of these known results are corollaries of the results in this paper. Additionally, our analytical approach based on linear programming duality makes it very easy to verify sufficiency proofs.
\end{abstract}

Index Terms- $f$-cast, Photonic Switching Fabrics, Crosstalk, Strictly Nonblocking

\section{INTRODUCTION}

Multicast-capable photonic switching architectures will likely play a central role in all-optical networking as most bandwidth-intensive applications require multicast support. Current multicast switch designs mostly focus on the broadcast case [1]-[3]. Although broadcast switches are certainly capable of supporting multicast with any fanout requirements, they are not scalable due to their prohibitively high hardware cost. Moreover, most multicast applications are restricted to a group of users, where broadcasting is rarely required. Hence, allocating expensive broadcast capability to each network switch is cost-inefficient for most practical purposes. Moreover, from the viewpoints of resource fairness and network security (e.g., limiting virus and worm propagation), we have other good reasons to impose a restriction on the maximum fanout of each request.

Consequently, there have been some recent research efforts on designing and analyzing $f$-cast switches, in which the maximum fanout of each request is upperbounded by the parameter $f$ [4]-[9]. An $f$-cast switch usually requires significantly lower hardware cost than its broadcast counterpart. (This point shall be illustrated in this paper with the $d$-ary multi-log architecture.) Furthermore, a good design of an $N \times N f$-cast switch covers both the unicast design $(f=1)$ and the broadcast

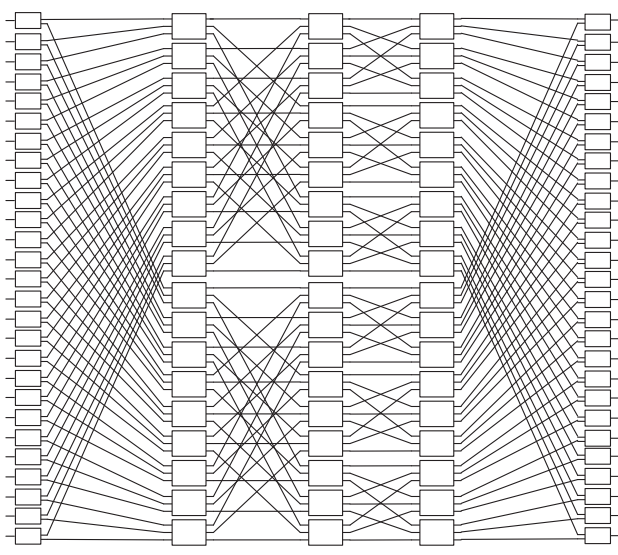

Fig. 1. Illustration of the $\log _{3}(27,0,2)$ network

design $(f=N)$ as special cases. Consequently, studying general $f$-cast switches is both mathematically pleasing and practically useful, as the results potentially can offer network designers more flexibility in selecting architectures for future multicast-intensive networks.

One of the major choices for switching architectures is the $d$-ary multilog network due to its small depth $(O(\log N))$, absolute signal loss uniformity, and good fault tolerance [9][16]. Henceforth, let $\log _{d}(N, 0, m)$ denote a $d$-ary multilog switch with $m$ vertically stacked inverse Banyan planes as shown in Figure 1.

When the multilog architecture is used to design a photonic switch, each switching element (SE) needs to be replaced by a functionally equivalent optical component. For instance, when $d=2$ we can use directional couplers as SEs [17]-[19]. However, directional couplers and many other optical switching elements suffer from optical crosstalk between interfering channels, which is one of the major obstacles in designing cost-effective switches [20]-[22]. To cope with crosstalk, the general crosstalk constraint was introduced by Vaez and Lea [11], which forces each new route to share at most $c$ SEs with other active routes in the switch, where $c$ is a parameter of the design. When $c=0$, no two routes are allowed to share a common SE, and we have a crosstalk-free switch [11], [14]. 
On the other hand, when $c=n=\log _{d} N$ a route can share any number of SEs with other routes (but no shared link), because each route in a $\log _{d}(N, 0, m)$ network contains exactly $n$ SEs. In this case only link-blocking takes effect, and it is commonly referred to as the link-blocking case, which is relevant to electronic switches [1], [2], [4]-[8], [12], [13], [15]. We shall refer to a switching network which is strictly nonblocking (SNB) under this general crosstalk constraint a $c$-SNB network. The reader unfamiliar with nonblocking concepts in switching networks is referred to [4].

For the first time in the literature, this paper derives conditions under which a $\log _{d}(N, 0, m)$ network is $f$-cast $c$-SNB. The problem setting is very general and thus challenging in many ways: (1) $f$-cast covers both unicast $(f=1)$ and multicast/broadcast $(f=N)$, (2) $d$-ary networks are much more general then the commonly considered binary multilog networks, and (3) the crosstalk constraint covers both the usual link-blocking case $(c=n)$ and the crosstalk-free case $(c=0)$.

Prior to our work, sufficient conditions for the multilog networks to be unicast $c$-SNB were derived in [11], [16]. In the $f$-cast SNB case, necessary and sufficient conditions are known for both link-blocking and crosstalk-free constraints [8], [9]. As we shall see, most of these known results are corollaries of the results in this paper. We will make use of the LP-duality method first proposed in [16] to analyze unicast networks. The $f$-cast case is considerably more complex, however.

The rest of the paper is organized as follows. Section II establishes basic notations and presents a simple algebraic view of $\log _{d}(N, 0, m)$ networks, which are used throughout the paper. Section III derives conditions under which $\log _{d}(N, 0, m)$ is $f$-cast $c$-SNB.

\section{PRELIMINARIES}

Throughout this paper, for any positive integers $k, d$, let $[k]$ denote the set $\{1, \ldots, k\}, \mathbb{Z}_{d}$ the set $\{0, \ldots, d-1\}$ which can be thought of as $d$-ary "symbols", $\mathbb{Z}_{d}^{k}$ the set of all $d$-ary strings of length $k$. Let $N=d^{n}$. We consider the $\log _{d}(N, 0, m)$ network, which denotes the stacking of $m$ copies of the $d$ ary inverse Banyan network $\mathrm{BY}^{-1}(n)$ with $N$ inputs and $N$ outputs. Label the inputs and outputs of $\mathrm{BY}^{-1}(n)$ with $d$-ary strings of length $n$. Specifically, each input $\mathbf{x} \in \mathbb{Z}_{d}^{n}$ and output $\mathbf{y} \in \mathbb{Z}_{d}^{n}$ have the form $\mathbf{x}=x_{1} \cdots x_{n}, \mathbf{y}=y_{1} \cdots y_{n}$, where $x_{i}, y_{i} \in \mathbb{Z}_{d}, \forall i \in[n]$. Also, label the $d \times d$ SEs in each of the $n$ stages of $\mathrm{BY}^{-1}(n)$ with $d$-ary strings of length $n-1$.

For any two $d$-ary strings $\mathbf{u}, \mathbf{v} \in \mathbb{Z}_{d}^{l}$, let $\operatorname{PRE}(\mathbf{u}, \mathbf{v})$ denote the longest common prefix, and $\operatorname{SUF}(\mathbf{u}, \mathbf{v})$ denote the longest common suffix of $\mathbf{u}$ and $\mathbf{v}$, respectively. For example, if $\mathbf{u}=0100110$ and $\mathbf{v}=0101010$, then $\operatorname{PRE}(\mathbf{u}, \mathbf{v})=010$ and $\operatorname{SUF}(\mathbf{u}, \mathbf{v})=10$. The following propositions were first observed in [9].

Proposition II.1. Let $(\mathbf{a}, \mathbf{b})$ and $(\mathbf{x}, \mathbf{y})$ be two unicast requests. Then their corresponding routes $R(\mathbf{a}, \mathbf{b})$ and $R(\mathbf{x}, \mathbf{y})$ in a $B Y^{-1}(n)$-plane share at least a common $S E$ if and only if

$\left|\operatorname{SUF}\left(\mathbf{a}_{1 . . n-1}, \mathbf{x}_{1 . . n-1}\right)\right|+\left|\operatorname{PRE}\left(\mathbf{b}_{1 . . n-1}, \mathbf{y}_{1 . . n-1}\right)\right| \geq n-1$.

Moreover, the routes $R(\mathbf{a}, \mathbf{b})$ and $R(\mathbf{x}, \mathbf{y})$ intersect at exactly one $S E$ if and only if

$\left|\operatorname{SUF}\left(\mathbf{a}_{1 . . n-1}, \mathbf{x}_{1 . . n-1}\right)\right|+\left|\operatorname{PRE}\left(\mathbf{b}_{1 . . n-1}, \mathbf{y}_{1 . . n-1}\right)\right|=n-1$,

in which case the common SE is an SE at stage numbered $\left|\operatorname{PRE}\left(\mathbf{b}_{1 . . n-1}, \mathbf{y}_{1 . . n-1}\right)\right|+1$ of the $B Y^{-1}(n)$-plane.

Proposition II.2. Let $(\mathbf{a}, \mathbf{b})$ and $(\mathbf{x}, \mathbf{y})$ be two unicast requests. Then their corresponding routes $R(\mathbf{a}, \mathbf{b})$ and $R(\mathbf{x}, \mathbf{y})$ in a $B Y^{-1}(n)$-plane share at least a common link if and only if

$$
\left|\operatorname{SUF}\left(\mathbf{a}_{1 . . n-1}, \mathbf{x}_{1 . . n-1}\right)\right|+\left|\operatorname{PRE}\left(\mathbf{b}_{1 . . n-1}, \mathbf{y}_{1 . . n-1}\right)\right| \geq n .
$$

\section{MAIN RESULTS}

The main idea is as follows. Consider a multicast request $(\mathbf{a}, B)$, where $\mathbf{a}$ is an input and $B$ is a subset of outputs. For each output $\mathbf{b} \in B$, the branch $(\mathbf{a}, \mathbf{b})$ can be routed independently from all other branches of the request. The number $\alpha$ of $\mathrm{BY}^{-1}(n)$-planes through which $(\mathbf{a}, \mathbf{b})$ cannot be routed through (those are called "blocking planes") can be expressed as the objective value of a feasible solution to a linear program (LP). Thus, by weak-duality $\alpha$ is upperbounded by the objective value $\beta$ of any feasible solution to the dualLP. Then, $m \geq \beta+1$ planes will be sufficient to route $(\mathbf{a}, \mathbf{b})$. This approach makes sufficiency proofs very easy to verify since they only involve checking if a solution is indeed dual-feasible. Of course, there is considerable effort "behind the scene" to discover good dual-feasible solutions because the linear programs are very general with many parameters $(n, c, f$, and $d)$ and variables.

Let $(\mathbf{a}, \mathbf{b})$ be an arbitrary branch of a multicast request. For each $i \in\{0, \ldots, n-1\}$, let

$$
A_{i}:=\left\{\mathbf{x} \in \mathbb{Z}_{d}^{n}-\{\mathbf{a}\} \mid \operatorname{SUF}\left(\mathbf{x}_{1 . . n-1}, \mathbf{a}_{1 . . n-1}\right)=i\right\} .
$$

Similarly, for each $j \in\{0, \ldots, n-1\}$, let

$$
B_{j}:=\left\{\mathbf{y} \in \mathbb{Z}_{d}^{n}-\{\mathbf{b}\} \mid \operatorname{PRE}\left(\mathbf{y}_{1 . . n-1}, \mathbf{b}_{1 . . n-1}\right)=j\right\} .
$$

Note that $\left|A_{i}\right|=\left|B_{i}\right|=d^{n-i}-d^{n-1-i}$, for all $0 \leq i \leq n-1$. Suppose the network $\log _{d}(N, 0, m)$ already had some ( $f$-cast) routes established. Consider a $\mathrm{BY}^{-1}(n)$ plane which blocks $(\mathbf{a}, \mathbf{b})$. There can only be three cases for which this happens: Case 1: There is a branch $(\mathbf{x}, \mathbf{y})$ of some $f$-cast request routed in the plane for which $R(\mathbf{x}, \mathbf{y})$ and $R(\mathbf{a}, \mathbf{b})$ share a link. By Proposition II.2, $(\mathbf{x}, \mathbf{y}) \in A_{i} \times B_{j}$ for some $i+j \geq n$. We will refer to $(\mathbf{x}, \mathbf{y})$ as a link-blocking branch.

Case 2: There is a branch $(\mathbf{x}, \mathbf{y})$ of some $f$-cast request whose route $R(\mathbf{x}, \mathbf{y})$ already intersects $c$ other routes at $c$ distinct SEs on the same plane, and adding $(\mathbf{a}, \mathbf{b})$ would introduce an additional intersecting $\mathrm{SE}$ to the route $R(\mathbf{x}, \mathbf{y})$. We will refer 
Maximize

$$
\sum_{i+j \geq n} x_{i j}+\sum_{i+j=n-1} y_{i j}+\frac{1}{c+1} \sum_{i+j=n-1} z_{i j}
$$

Subject to

$$
\begin{aligned}
& \sum_{j: i+j \geq n} x_{i j}+\sum_{j: i+j=n-1}\left(y_{i j}+z_{i j}\right)+\sum_{j: i+j<n-1}\left(l_{i j}+r_{i j}\right) \leq f\left(d^{n-i}-d^{n-1-i}\right) \quad \forall i \\
& \sum_{i: i+j \geq n} x_{i j}+\sum_{i: i+j=n-1}\left(y_{i j}+z_{i j}\right)+\sum_{i: i+j<n-1}\left(l_{i j}+r_{i j}\right) \leq d^{n-j}-d^{n-1-j} \quad \forall j \\
& z_{i j} \leq \frac{1}{c+1} \sum_{i^{\prime}+j^{\prime}=n-1} z_{i^{\prime} j^{\prime}} \quad i+j=n-1 \\
& c y_{i j}=\left(\sum_{i^{\prime}<i} r_{i^{\prime} j}+\sum_{j^{\prime}<j} l_{i j^{\prime}}\right) \quad i+j=n-1 \\
& l_{i j^{\prime}} \leq \quad y_{i j} \quad i+j=n-1, j^{\prime}<j \\
& r_{i^{\prime} j} \leq \quad y_{i j} \quad i+j=n-1, i^{\prime}<i \\
& x_{i j}, y_{i j}, z_{i j}, l_{i j}, r_{i j}, p_{i} \geq \quad 0 \quad \forall i, j
\end{aligned}
$$

\section{The Dual LP}

Minimize

$$
\sum_{i=0}^{n-1} f\left(d^{n-i}-d^{n-1-i}\right) u_{i}+\sum_{j=0}^{n-1}\left(d^{n-j}-d^{n-1-j}\right) v_{j}
$$

Subject to

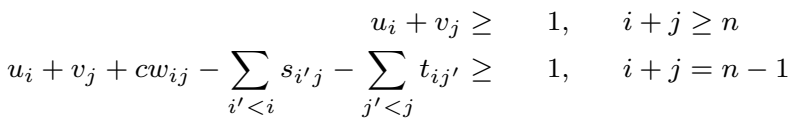

$$
\begin{aligned}
& u_{i}+v_{j}+\frac{c}{c+1} p_{i}-\frac{1}{c+1} \sum_{i^{\prime} \neq i} p_{i^{\prime}} \geq \quad \frac{1}{c+1}, \quad i+j=n-1 \\
& u_{i}+v_{j}-w_{i, n-1-i}+t_{i j} \geq \quad 0, \quad i+j<n-1 \\
& u_{i}+v_{j}-w_{n-1-j, j}+s_{i j} \geq 0, \quad i+j<n-1 \\
& u_{i}, v_{j}, s_{i j}, t_{i j} \geq \quad 0, \quad \forall i, j
\end{aligned}
$$

Fig. 2. The Primal and the Dual Linear Programs

to these $c$ other branches as secondary branches accompanying $(\mathbf{x}, \mathbf{y})$, and to $(\mathbf{x}, \mathbf{y})$ as a node-blocking branch of type 1 .

By Proposition II.1 $(\mathbf{x}, \mathbf{y}) \in A_{i} \times B_{j}$ for some $i+j=n-1$. Moreover, the common SE between $R(\mathbf{a}, \mathbf{b})$ and $R(\mathbf{x}, \mathbf{y})$ is at stage $j+1$. The routes for secondary branches must thus intersect $R(\mathbf{x}, \mathbf{y})$ at stages strictly less than $j+1$ or strictly greater than $j+1$.

If a secondary branch $(\mathbf{u}, \mathbf{v})$ has its route intersects $R(\mathbf{x}, \mathbf{y})$ at stage $1 \leq s<j+1$, it follows that $\operatorname{PRE}(\mathbf{y}, \mathbf{v})=s-1<j$, and $\operatorname{SUF}(\mathbf{x}, \mathbf{u})=n-1-(s-1)=n-s>n-1-j=i$. Hence, $(\mathbf{u}, \mathbf{v}) \in A_{i} \times B_{s-1}$. We will refer to such $(\mathbf{u}, \mathbf{v})$ as a left secondary branch.

If a secondary branch $(\mathbf{u}, \mathbf{v})$ has its route intersects $R(\mathbf{x}, \mathbf{y})$ at stage $j+1<s \leq n$, it follows that $\operatorname{PRE}(\mathbf{y}, \mathbf{v})=s-1>j$, and $\operatorname{SUF}(\mathbf{x}, \mathbf{u})=n-1-(s-1)=n-s<n-1-j=i$. Hence, $(\mathbf{u}, \mathbf{v}) \in A_{n-s} \times B_{j}$. We will refer to such $(\mathbf{u}, \mathbf{v})$ as a right secondary branch.

To summarize, there are two types of secondary branches accompanying $(\mathbf{x}, \mathbf{y})$ : the left secondary branches are the branches $(\mathbf{u}, \mathbf{v}) \in A_{i} \times B_{j^{\prime}}$ for some $j^{\prime}<j$, and the right secondary branches are the branches $(\mathbf{u}, \mathbf{v}) \in A_{i^{\prime}} \times B_{j}$ for some $i^{\prime}<i$. For each $i^{\prime}<i$ there is at most one right secondary branch in $A_{i^{\prime}} \times B_{j}$. Similarly, for each $j^{\prime}<j$ there is at most one left secondary branch in $A_{i} \times B_{j^{\prime}}$.

Case 3: There are $c+1$ branches (of some $f$-cast requests) in the plane each of whose routes intersects $(\mathbf{a}, \mathbf{b})$ at exactly one SE at a distinct stage. These will be called node-blocking requests of type 2 . If $(\mathbf{x}, \mathbf{y})$ is such a request, then $(\mathbf{x}, \mathbf{y}) \in$ $A_{i} \times B_{j}$ for some $i+j=n-1$. Moreover, two node-blocking branches of type 2 belonging to the same $A_{i} \times B_{j}$ must belong to different blocking planes.

Theorem III.1. The number of blocking planes for $(\mathbf{a}, \mathbf{b})$ is the objective value of a feasible solution to the primal linear program as shown in Figure 2.

Proof: For each pair $i+j \geq n$, let $x_{i j}$ be the number of link-blocking branches in $A_{i} \times B_{j}$. For each $i+j=n-1$, let $y_{i j}$ be the number of node-blocking branches of type 1 , and $z_{i j}$ be the number of node-blocking branches of type 2 in $A_{i} \times B_{j}$. For each pair $i+j<n-1$, let $l_{i j}$ and $r_{i j}$ be the 
number of left and right secondary branches in $A_{i} \times B_{j}$. The number of blocking planes is thus expressed in the objective function (4).

We next explain why the variables satisfy all the constraints. Recall that $\left|A_{i}\right|=d^{n-i}-d^{n-1-i}, \forall i$. Thus, the number of branches of $f$-cast requests out of $A_{i}$ is at most $f\left(d^{n-i}-\right.$ $\left.d^{n-1-i}\right)$, justifying constraint (5). Similarly, bounding the number of branches of requests into $B_{j}$ explains constraint (6). Since all node-blocking branches of type 2 belonging to the same $A_{i} \times B_{j}$ must belong to different blocking planes, $z_{i j}$ is at most the number of blocking planes involving these type2 branches. This is constraint (7). Constraint (8) expresses the fact that, for every node-blocking request of type 1 in $A_{i} \times B_{j}$ $(i+j=n-1)$, there must be $c$ accompanying secondary requests (left or right). Lastly, for each node-blocking request of type 1 in $A_{i} \times B_{j}(i+j=n-1)$ constraint (8) says that for each $j^{\prime}<j$ there is at most one left secondary request in $A_{i} \times B_{j^{\prime}}$, and constraint (10) says that for each $i^{\prime}<i$ there is at most one right secondary request in $A_{i^{\prime}} \times B_{j}$.

The dual of the primal LP is also given in Figure 2. As an illustration of the power of our method, let us reproduce a few known results. The corollaries should give the reader the correct insight without delving into too much technicality.

The following condition has been shown in [9] to be necessary and sufficient, with a fairly complex combinatorial argument.

Corollary III.2 (The crosstalk-free case). For $\log _{d}(N, 0, m)$ to be $f$-cast crosstalk-free $S N B$, it is sufficient that

$$
m \geq f\left(d^{\left\lceil\frac{n-r-1}{2}\right\rceil}-1\right)+d^{n-\left\lceil\frac{n-r-1}{2}\right\rceil} .
$$

Proof: Being crosstalk-free SNB is the same as being 0SNB (i.e. $c=0$ ). Let $b=\left\lceil\frac{n-r-1}{2}\right\rceil$, and $a=n-b$. Consider the following assignment to the dual variables: $u_{i}=1, \forall i \geq a$, $v_{j}=1, \forall j \geq b$, and all other variables are 0 . This solution is dual-feasible with objective value

$$
f\left(d^{\left\lceil\frac{n-r-1}{2}\right\rceil}-1\right)+d^{n-\left\lceil\frac{n-r-1}{2}\right\rceil}-1,
$$

which is an upperbound on the number of blocking planes. One more plane is needed for the network to be SNB, which completes the proof.

The following condition has been shown in [8], [9] to be necessary and sufficient for the link-blocking case - again using a complex combinatorial argument. Our proof is much simpler.

Corollary III.3 (The link-blocking case). Define

$$
m_{\mathrm{lb}}(n, f, d):=f\left(d^{\left\lceil\frac{n-r-2}{2}\right\rceil}-1\right)+d^{n-\left\lceil\frac{n-r}{2}\right\rceil} .
$$

For $\log _{d}(N, 0, m)$ to be $f$-cast SNB in the link-blocking sense, it is sufficient that

$$
m \geq \begin{cases}m_{\mathrm{lb}}(n, f, d) & f<d^{n-2} \\ m_{\mathrm{lb}}\left(n, d^{n-2}, d\right) & f \geq d^{n-2}\end{cases}
$$

Proof: First, suppose $f<d^{n-2}$. Let $b=\left\lceil\frac{n-r}{2}\right\rceil$, and $a=n+1-b$. Consider the following assignment to the dual variables: $u_{i}=1, \forall i \geq a, v_{j}=1, \forall j \geq b, w_{a-1, n-a}=1$, $p_{a-1}=1 / c, s_{i, b-1}=t_{a-1, j}=1$ when $i<a-1, j<b-1$ and all other variables are 0 . This solution is dual-feasible with objective value precisely $m_{\mathrm{lb}}(n, f, d)-1$. Second, when $f \geq d^{n-2}$, assign all $v_{j}=1, j>0, w_{n-1,0}=1, p_{n-1}=1 / c$, $s_{i, 0}=1, \forall i<n-1$ and all other variables to be 0 .

Henceforth, we consider the case when $1 \leq c \leq n-1$. In the next three lemmas, we derive several general dual-feasible solutions. Then, depending on the relative relationships between $f, n, c, d$ we apply one of the lemmas to get the best sufficient condition.

Lemma III.4. Suppose $1 \leq c \leq n-1$. Let $a, b, p, q$ be any integers satisfying $0 \leq a \leq n-1, b=n-1-a, 0 \leq p \leq b$, $0 \leq q \leq a$. Define $\delta=\frac{1}{d(c+1)}$ and $\lambda=\frac{d}{c+1}$. Then, there exists a dual-feasible solution with objective value

$$
\begin{aligned}
f\left(d^{b}-1\right)+ & \left(d^{a}-1\right)-\frac{(d-1)\left[f d^{b}\left(1-\delta^{p}\right)+d^{a}\left(1-\delta^{q}\right)\right]}{d(c+2)(1-\delta)} \\
+ & \frac{(d-1)}{c+2}\left[f d^{b} \frac{1-\lambda^{q+1}}{1-\lambda}+d^{a} \frac{1-\lambda^{p+1}}{1-\lambda}\right] .
\end{aligned}
$$

We agree on the convention that, for any integer $k, \frac{\left(1-\lambda^{k}\right)}{(1-\lambda)}=k$ when $\lambda=1$.

Proof: Consider the following assignment to the dual variables: $u_{i}=\frac{1}{(c+2)(c+1)^{a-i}}$ when $a-q \leq i \leq a$, $u_{i}=1-\frac{1}{(c+2)(c+1)^{i-(a+1)}}$ when $a+1 \leq i \leq a+p$, $u_{i}=1$ when $a+p+1 \leq i \leq n-1, v_{j}=\frac{1}{(c+2)(c+1)^{b-j}}$ when $b-p \leq j \leq b, v_{j}=1-\frac{1}{(c+2)(c+1)^{j-(b+1)}}$ when $b+1 \leq j \leq b+q, v_{j}=1$ when $b+q+1 \leq j \leq n-1$, $w_{i j}=\min \left\{u_{i}, u_{j}\right\}$ for all $i+j=n-1$. All other variables are 0 .

To this end, we verify that this solution is indeed dual feasible. Constraints (13) and (15) can be verified straightforwardly. Constraints (16) and (17) follow from the fact that $u_{i} \geq w_{i, n-1-i}$, and $v_{j} \geq w_{n-1-j, j}$.

Next, consider the dual constraint (14). When $i \leq a-q-1$ or $i \geq a+p+1$, we have $v_{j}=1$ or $u_{i}=1$ (recall $i+j=n-1$ ). The constraint is satisfied because in these cases $w_{i j}=0$. When $i=a, u_{i}+v_{j}+c w_{i j}=1$. When $a+1 \leq i=a+k \leq$ $a+p$, we have

$$
\begin{aligned}
u_{i}+v_{j}+c w_{i j} & =1-\frac{1}{(c+2)(c+1)^{k-1}} \\
& +\frac{1}{(c+2)(c+1)^{k}}+\frac{c}{(c+2)(c+1)^{k}}=1
\end{aligned}
$$

When $a-q \leq i=a-k \leq a-1$, the constraint is verified similarly.

Lemma III.5. Suppose $c \geq \frac{n}{2}+1$. Let a be any integer where $n-c+1 \leq a \leq c-1$, and $x$ be any real number in the interval $\left[\frac{1}{c-a+1}, \frac{c+a-n}{c+a-n+1}\right]$. Then, there exists a feasible solution to the dual LP with objective value

$$
f\left(d^{n-a-1}-1\right)+\left(d^{a}-1\right)+(d-1) d^{n-a+1} x\left(f-d^{2 a-n}\right) .
$$


Proof: Consider the following assignment to the dual variables: $u_{a}=x, v_{n-a}=1-x, u_{i}=v_{j}=1$ when $i>a$ and $j>n-a, w_{a, n-1-a}=s_{i, n-1-a}=\frac{1}{c_{1} a+1}$ when $0 \leq i \leq a-1$, and $w_{a-1, n-1}=t_{a-1, j}=\frac{1}{c+a-n+1}$ when $0 \leq j \leq n-a-1$. All other variables are 0 .

Lemma III.6. Suppose $c \geq \frac{n}{2}+1$. Let a be any integer where $n-c+1 \leq a \leq c-1$. There exists a dual-feasible solution with objective value

$f\left(d^{n-a-1}-1\right)+\left(d^{a}-1\right)+\frac{1}{c+2} d^{a-1}(d-1)^{2}\left(f d^{n-2 a-1}+1\right)$.

Proof: Consider the following assignment to the dual variables: $u_{a}=v_{n-1-a}=\frac{1}{c+2}, u_{a+1}=v_{n-a}=\frac{c+1}{c+2}$, $u_{i}=v_{j}=1$ when $i>a, j>n-a, w_{a-1, n-a}=$ $t_{a_{1}, j}=\frac{1}{(c+2)(c+a+1-n)}$ where $j \leq n-a-2, w_{a}=\frac{1}{c+2}$, $w_{a+1}=s_{i, n-a-2}=\frac{1}{(c+2)(c-a)}$ when $i \leq a-1$. All other variables are 0 .

Applying Lemma III.4 with $a=\bar{a}, b=\bar{b}, p=\bar{p}$, and $q=\bar{q}$ as defined below, we obtain the following theorem. The results for $c \leq n / 2$ in [11], [16] are easily seen to be consequences of this theorem for $f=1$. In fact, our result is even slightly better!

Theorem III.7. Consider the case when $c \leq \frac{n}{2}$. Let $\bar{a}=$ $\left\lfloor\frac{n+r}{2}\right\rfloor, \bar{b}=n-1-\bar{a}, \bar{p}=\left\lfloor\frac{1}{2} \log _{d} f(c+1)+\frac{n-1}{2}-\bar{a}\right\rfloor$, $\bar{q}=\left\lfloor\frac{1}{2} \log _{d} \frac{c+1}{f}+\bar{a}-\frac{n-1}{2}\right\rfloor, \delta=\frac{1}{d(c+1)}$, and $\lambda=\frac{d}{c+1}$. Then,

$$
\begin{aligned}
m \geq f\left(d^{\bar{b}}-1\right) & +d^{\bar{a}}-\frac{(d-1)\left[f d^{\bar{b}}\left(1-\delta^{\bar{p}}\right)+d^{\bar{a}}\left(1-\delta^{\bar{q}}\right)\right]}{d(c+2)(1-\delta)} \\
& +\frac{(d-1)}{c+2}\left[f d^{\bar{b}} \frac{1-\lambda^{\bar{q}+1}}{1-\lambda}+d^{\bar{a}} \frac{1-\lambda^{\bar{p}+1}}{1-\lambda}\right]
\end{aligned}
$$

is sufficient for $\log _{d}(N, 0, m)$ to be $f$-cast $c$-SNB. (Again, set $\frac{\left(1-\lambda^{k}\right)}{(1-\lambda)}=k$ when $\lambda=1$.)

Applying Lemmas III.5 and III.6 appropriately (after some tedious but straightforward calculus) we obtain the following theorem. Again, the results for $c>n / 2$ in [11], [16] consequences of this theorem for $f=1$.

Theorem III.8. Consider the case when $c \geq \frac{n}{2}+1$. Set $\bar{a}=$ $\lceil(n+r) / 2\rceil$. Note that in this case $n-c+1 \leq \bar{a}$.

(i) If $\bar{a} \leq c-1$ and $n+r$ is even, then

$$
m \geq f\left(d^{n-\bar{a}-1}-1\right)+d^{\bar{a}}+\frac{(d-1) d^{n-\bar{a}+1}\left(f-d^{2 \bar{a}-n}\right)}{c-\bar{a}+1}
$$

is sufficient for the network to be f-cast c-SNB.

(ii) If $\bar{a} \leq c-1$ and $n+r$ is odd, then

$$
\begin{aligned}
& m \geq f\left(d^{n-\bar{a}-1}-1\right)+d^{\bar{a}}+ \\
& \frac{(d-1) d^{n-\bar{a}+1}\left(f-d^{2 \bar{a}-n}\right)(c+\bar{a}-n)}{c+\bar{a}-n+1}
\end{aligned}
$$

is sufficient for the network to be f-cast c-SNB.

(iii) If $\bar{a}>c-1$, then

$$
m \geq f\left(d^{n-\bar{a}}-1\right)+d^{\bar{a}-1}+\frac{d^{\bar{a}-2}(d-1)^{2}\left(f d^{n-2 \bar{a}+1}+1\right)}{c+2}
$$

is sufficient for the network to be f-cast c-SNB.

\section{REMARKS}

We have had to omit quite a lot of technical details due to space limitation. However, we believe that the general approach and intuition were presented clear enough for the reader to get the basic line of thought.

\section{REFERENCES}

[1] W. Kabacinski and G. Danilewicz, "Wide-sense and strict-sense nonblocking operation of multicast multi- $\log _{2} n$ switching networks," IEEE Transactions on Communications, vol. 50, pp. 1025-1036, Jun 2002.

[2] F.K.Hwang and B.-C. Lin, "Wide-sense nonblocking multicast $\log _{2}(n, m, p)$ networks," IEEE Transactions on Communications, vol. 51, pp. 1730-1735, Oct 2003.

[3] Y. Yang and G. M. Masson, "Nonblocking broadcast switching networks," IEEE Trans. Comput., vol. 40, no. 9, pp. 1005-1015, 1981.

[4] F. K. Hwang, The mathematical theory of nonblocking switching networks. River Edge, NJ: World Scientific Publishing Co. Inc., 2004.

[5] H.-B. Chen and F. K. Hwang, "On multicast rearrangeable 3-stage clos networks without first-stage fan-out," SIAM Journal on Discrete Mathematics, vol. 20, no. 2, pp. 287-290, 2006.

[6] A. Pattavina and G. Tesei, "Non-blocking conditions of multicast threestage interconnection networks," IEEE Transactions on Communications, vol. 46, pp. 163-170, Dec 2005.

[7] X. Jiang, A. Pattavina, and S.Horiguchi, "Rearrangeable $f$-cast multi$\log 2 n$ networks," IEEE Transactions on Communications, 2007. to appear.

[8] F.K.Hwang, Y. Wang, and J. Tan, "Strictly nonblocking f-cast $\log _{d}(n, m, p)$ networks," IEEE Transactions on Communications, vol. 55, pp. 981-986, May 2007.

[9] Y. Wang, H. Q. Ngo, and X. Jiang, "Strictly nonblocking $f$-cast $d$-ary multilog networks under fanout and crosstalk constraints," in Proceedings of the 2008 International Conference on Communications (ICC), (Bejing, China), IEEE, 2008.

[10] C.-T. Lea, "Muti- $\log _{2} n$ networks and their applications in high speed electronic and photonic switching systems," IEEE Transactions on Communications, vol. 38, no. 10, pp. 1740-1749, 1990.

[11] M. Vaez and C.-T. Lea, "Strictly nonblocking directional-coupler-based switching networks under crosstalk constraint," IEEE Trans. Comm., vol. 48, pp. 316-323, Feb 2000.

[12] F. Hwang, "Choosing the best $\log _{2}(n, m, p)$ strictly nonblocking networks," IEEE Transactions on Communications, vol. 46, pp. 454-455, Dec 1998.

[13] D.-J. Shyy and C.-T. Lea, " $\log _{2}(n, m, p)$ strictly nonblocking networks," IEEE Transactions on Communications, vol. 39, no. 10, pp. 1502-1510, 1991.

[14] G. Maier and A. Pattavina, "Design of photonic rearrangeable networks with zero first-order switching-element-crosstalk," IEEE Trans. Comm., vol. 49, pp. 1248-1279, Jul 2001.

[15] C.-T. Lea and D.-J. Shyy, "Tradeoff of horizontal decomposition versus vertical stacking in rearrangeable nonblocking networks," IEEE Transactions on Communications, vol. 39, pp. 899-904, 1991.

[16] H. Q. Ngo, Y. Wang, and A. Le, "A linear programming duality approach to analyzing strictly nonblocking $d$-ary multilog networks under general crosstalk constraints," in Proceedings of the 14th Annual International Computing and Combinatorics Conference (COCOON), (Bejing, China), Springer, LNCS, 2008.

[17] T. E. Stern and K. Bala, Multiwavelength Optical Networks: A Layered Approach. Upper Saddle River, NJ: Prentice Hall PTR, 1999.

[18] J.-C. Wu and T.-L. Tsai, "Low complexity design of a wavelengthselective switch using raman amplifiers and directional couplers," in GLOBECOM, 2006.

[19] B. Mukherjee, Optical Communication Networks. New York, NY: McGraw-Hill, 1997.

[20] M. Vaez and C.-T. Lea, "Wide-sense nonblocking Banyan-type switching systems based on directional couplers," IEEE J. Select. Areas Commun., vol. 16, pp. 1327-1332, Sep 1998.

[21] V. Chinni, T. Huang, P.-K. Wai, C. Menyuk, and G. Simonis, "Crosstalk in a lossy directional coupler switch," J. Lightwave Technol., vol. 13, no. 7, pp. 1530-1535, 1995.

[22] D.Li, "Elimination of crosstalk in directional coupler switches," Optical Quantum Electron.,, vol. 25, no. 4, pp. 255-260, 1993. 